\title{
CHARACTERIZATION AND MODELING OF SOLITON TRANSMISSION AT 2.5 GB/S OVER $200 \mathrm{KM}$
}

\author{
Khalid A. S. AL-Khateeb, Mohd ARmi, and Mohamad Shawal \\ Department of Electrical and Computer Engineering \\ Kulliyyah of Engineering, International Islamic University Malaysia, \\ Jalan Gombak, 53100 Kuala Lumpur, MALAYSIA \\ E-mail: khalid@iiu.edu.my
}

\begin{abstract}
Soliton characteristics and soliton transmission have been simulated using a VPI simulator. Simulation was also used to construct and study a soliton communication system. Near soliton pulses emitted by an actively mode-locked laser is then compressed in a dispersion-compensating fibcr (DCF) to produce solitons. The effects of non-linearity and active pre-chirping of mode-locked laser diode sources were also investigated. Assessment on a modeled system using real data shows that propagation over $250 \mathrm{~km}$ at $2.5 \mathrm{~Gb} / \mathrm{s}$ in standard fibers with $20 \mathrm{ps}$ pulse widths is possible in the $1550 \mathrm{~nm}$ wavelength range.
\end{abstract}

Keywords: Optical communications, solitons, dispersion compensation, long haul transmission

\section{INTRODUCTION}

Modern telecommunication systems around the world have been revolutionized by fiber optics (FO). This has enabled an enormous amount of information to be exchanged within extremely short periods of time. There are more than 40 million kilometers of optical fibers installed worldwide [1]. Most of which are standard fibers with low-loss in the $1310 \mathrm{~nm}$ and $1550 \mathrm{~nm}$ wavelength regions. These two spectral regions of low absorption are called the second and third window, respectively [2]. Most of the current systems work in the second window. In order to maintain the viability of the data over the length of the communication system, electronic regenerators are used to retime, reshape and amplify the optical signals periodically. These regenerators however, present a major drawback. The data rate in which they operate is somewhat limited. Interest has therefore turned to replacing these regenerators with erbium-doped fiber amplifiers (EDFAs), which are wide-bandwidth amplifiers. These amplifiers on the other hand operate mostly in the third communications window, $1550 \mathrm{~nm}$ range [1]. This has stimulated more research in this area including the use of long haul solitons, the subject of the current work.

Solitons are a unique phenomenon of maintaining a reasonably constant pulse-width, even after they travel over extremely long distances. They were first observed in water waves traveling in canals. These pulses are also observed in light waves traveling along optical fibers, which is what makes them of great interest in telecommunications. 
An important feature is that solitons are robust in the presence of various perturbations (e.g. loss or non-perfect launch conditions). The perturbed pulses usually evolve as stable solitons. Canceling the effect of group velocity delay (GVD) can be achieved in conventional systems by using periodically placed dispersion-compensating sections of fibers [3]. There are several features of solitons which make them suitable as carriers of high capacity data. Of course, there are also limitations, as in any engineering system. In solitons the limitations are caused by nonlinear interactions, such as dispersive waves, and various types of timing jitter [4].

\section{SYSTEM MODELING}

The system model is based on the implementation of the soliton pulses in a $22 \mathrm{~km}$ span of fiber using dispersion compensation method. The model shows that solitons can propagate up to $250 \mathrm{~km}$ at $2.5 \mathrm{~Gb} / \mathrm{s}$ without the need of regenerators.

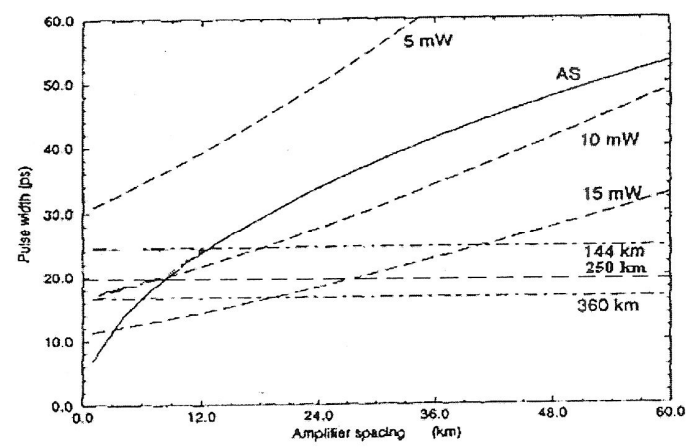

Fig. 1: Design diagram for amplifier spacing and average power constraints.

Both dispersion compensation and pre-chirping were taken into consideration by the simulation program [4]. The average dispersion $D_{a}$ [5], of a section of standard fiber with dispersion $D_{s}$ and length $z_{s}$, concatenated with a compensating fiber of dispersion $D_{c}$ and length $z_{c}$, is given by:

$$
D_{a}=\frac{\left(D_{S} z_{S}+D_{C} z_{C}\right)}{z_{S}+z_{C}}
$$

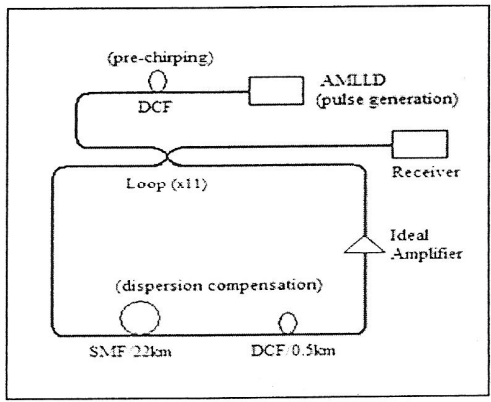

Fig. 2: System modeling. 
The model of the system is shown in Fig. 2. It consists of an actively mode-locked laser diode (AMLLD) source, operating at $1550 \mathrm{~nm}$ generates $19.7 \mathrm{ps}$ soliton pulses at $5 \mathrm{GHz}$. Pre-chirping is achieved using a short length of dispersion compensated fiber (DCF) of $D=-150 \mathrm{ps} / \mathrm{nm} / \mathrm{km}$. The soliton pulse is launched in a re-circulation loop which is made of a $22 \mathrm{~km}$ span of standard mode fiber (SMF) with $D=6 \mathrm{ps} / \mathrm{nm} / \mathrm{km}$, followed by a $0.5 \mathrm{~km}$ of DCF with $\mathrm{D}=-90 \mathrm{ps} / \mathrm{nm} / \mathrm{km}$.

\section{DISPERSION COMPENSATION SCHEME}

Dispersion compensation is one of the methods of dealing with pulse dispersion. For soliton transmission systems, this method makes use of the nonlinearity of the optical fiber to counter the dispersion. The compensation scheme usually involves two sections of fiber, one with positive dispersion followed by one with negative dispersion, such that the two effects cancel each other, as shown in Fig.3. In positive dispersion, the longer wavelengths travel faster than the shorter wavelengths, whereas in the second it is the other way round. Hence, the spreading of the pulses in the first section is turned into compression in the second.

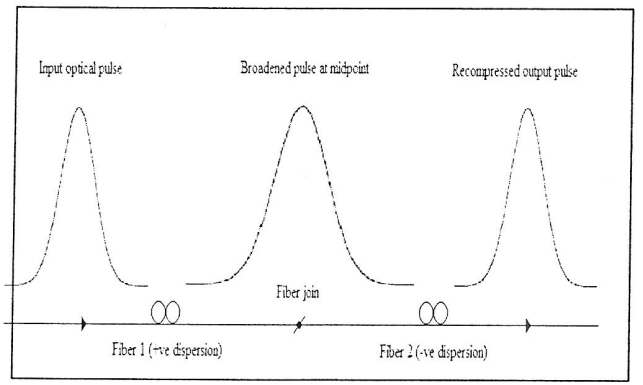

Fig. 3: Dispersion management system.

\section{SOLITON SYSTEM DESIGN}

The design of a soliton communications system requires consideration of several factors, such as soliton width, soliton spacing, and the distance between successive amplification stages. The input pulse should have a hyperbolic secant profile in order to propagate as a fundamental soliton inside the optical fiber.

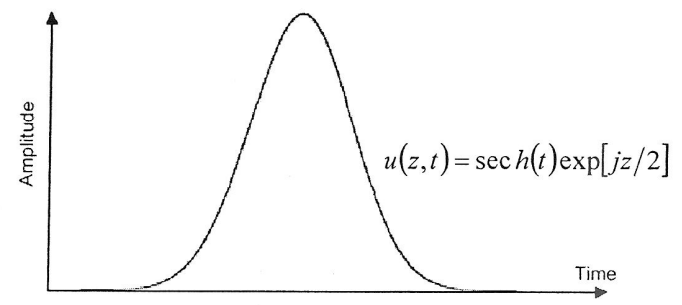

Fig. 4: Fundamental Soliton Pulse. 
The field amplitude at the fiber input is given by [1];

$$
\mathrm{A}(0, \mathrm{t})=\sqrt{\mathrm{P}_{0}} \operatorname{sech}\left(\mathrm{t} / \mathrm{T}_{\mathrm{s}}\right) \ldots
$$

where $P_{0}$ is the peak power of the pulse, and $T_{s}$ is the full-width at half maximum power (FWHM) of the soliton pulse.

To derive the evolution of the pulse shape required for soliton transmission, the nonlinear Schrödinger equation (NLS) must be considered [5]. For the solution of NLS, the power is given by the square of the envelop function. Thus $T_{\mathrm{s}}$ of the fundamental soliton pulse in normalized time is found from the relationship $\operatorname{sech}^{2}(\tau)=1 / 2$, with $\tau=T_{s} /\left(2 T_{0}\right)$, and $T_{0}$ is the basic normalized time unit given by [5]:

$$
\mathrm{T}_{\mathrm{o}}=\mathrm{T}_{\mathrm{s}} /\left(2 \cosh ^{-1} \sqrt{ } 2\right)=\mathrm{T}_{\mathrm{s}} / 1.762 \ldots \ldots
$$

The normalized distance parameter or the dispersion length $L_{\text {disp }}$ is a measure of the period of a soliton. This parameter is given by [5]:

$$
\mathrm{L}_{\text {disp }}=2 \pi \mathrm{c} \mathrm{T}_{0}^{2} /\left(\lambda^{2} \mathrm{D}\right)=\left(1 /\left[2 \cosh ^{-1} \sqrt{2}\right]^{2}\right)\left(2 \pi \mathrm{c} \mathrm{T}{ }_{\mathrm{s}}^{2} /\left(\lambda^{2} \mathrm{D}\right) \ldots\right.
$$

Where $c$ is the speed of light, $\lambda$ is the wavelength in vacuum and $D$ is the dispersion of the fiber. The soliton period is defined as the distance over the Kerr-induced phase shift. Numerical simulations indicate that a stable soliton can be transmitted over a distance of $10,000 \mathrm{~km}$, as long as $L_{\text {disp }}>5 L$, where $L_{\text {disp }}$ is related to the soliton period and $L$ is the amplifier spacing.

The amplifier spacing $L$ plays a role analogous to the repeater spacing in conventional transmission systems. Ideally, $L$ should be as large as possible in order to minimize the overall system cost. The amplifier spacing can be as large as $100 \mathrm{~km}$ for non-soliton $1550 \mathrm{~nm}$ systems making use of Erbium Doped Fiber Amplifiers (EDFAs). For soliton communications systems, the optimum value of $L$ is generally smaller and depends on many parameters. Soliton can be propagated over a few thousands kilometers provided that the amplifier spacing $L$ is kept considerably below the soliton period $T_{0}[6]$.

However, for long haul fiber link, the fiber loss causes the attenuation of soliton power, resulting in the destruction of the soliton waveform. In order to transmit the soliton in such longhaul link, the lumped optical amplifiers are inserted periodically. The soliton is amplified in such a way that the average power in the amplifier span is equal to the value required for ideal soliton. This concept is called average soliton [7].

Therefore, a practical system can be designed if the amplifier spacing is large enough. However, the calculations can be void if the simulation is not implemented properly. It should be noted that optical amplifiers add noise to the signal in the form of amplified spontaneous emission (ASE). This noise affects the performance in ways that degrade the signal-to-noise ratio (SNR). The extent of degradation depends among other things, on the amplifier noise figure and the number of amplifiers in the system [2]. The effect of the amplified spontaneous emission (ASE) can also lead to gain-saturation. The dielectric response to electromagnetic waves is typically frequency dependent. This results in the dependence of the index of refraction on frequency. There are also certain natural frequencies at which absorption takes place in the material. Since the speed of a wave depends on the index of refraction, a wave composed of several harmonics will tend to disperse. This chromatic dispersion tends to distort the incoming signals. The dispersive characteristics of a fiber can be minimized in the operating region of the system by changing the core radius and the difference in the refractive indices between the core and the cladding. 
The main parameter that characterizes dispersion is the rate of change of the group velocity dispersion with respect to the frequency [8]. The fiber exhibits positive dispersion, or $\beta_{1}$ normal GVD for longer wavelengths, and negative dispersion, or $\beta_{2}$ anomalous GVD.

The anomalous parameter of GVD can be written as:

$$
\beta_{2}=-\frac{\lambda^{2} D}{2 \pi c}
$$

These determine the amount of broadening that an impulse experiences as it propagates inside a fiber. In order to understand the amplification of solitons and soliton interaction, it is important to try solving the non-linear Schrödinger equation (NLS) with the input amplitude consisting of a soliton, so that:

$$
U(0, \tau)=\operatorname{sech}\left(\tau-q_{0}\right)+r \operatorname{sech}\left[r\left(\tau+q_{0}\right)\right] \exp (j \theta)
$$

Where $r$ is the relative amplitude of the two solitons, $\theta$ is the relative phase, and $2 q_{0}$ is the initial (normalized) separation. If the soliton pair represents two solitons in neighboring bit slots, their separation $2 q_{0}, T_{0}$ should equal $T_{B}$. By using $B=1 / T_{B}, q_{0}$ is related to the bit rate as;

$$
B=\frac{1}{2 q_{0} T_{0}}=\frac{0.88}{q_{0} T_{F W H M}}
$$

Therefore, the dispersion compensation technique is an interesting method of soliton transmission. Finally the average value of $P_{0}$ for the soliton along the path can be calculated as follows;

$$
P_{0}-\frac{0.776 \lambda^{3} A_{\text {eff }} D}{T_{F W H M} \pi^{2} c n_{2}}
$$

where $A_{e f f}$ is the effective core area and $n_{2}$ is the nonlinear coefficient.

\section{RESULTS}

Assuming a dispersion shifted fiber [5] having $D=0.5 \mathrm{ps} / \mathrm{nm} . \mathrm{km}$, at $\lambda=1550 \mathrm{~nm}$, for a pulse of FWHM, $T_{s}=20 \mathrm{ps}$, using equation (4), the dispersion length will be $202 \mathrm{~km}$, i.e. on the order of hundreds of kilometers.

Simulations were performed to test the maximum transmission distance for which data can be recovered. Results show that soliton pulses of $19.7 \mathrm{ps}$ can propagate to $250 \mathrm{~km}$ without deterioration, after which the pulse train begins to distort, as shown in Fig 5. At $250 \mathrm{~km}$, although the pulse shapes appear to have been corrupted, the energy associated with each data bit remains in the appropriate bit slot. 


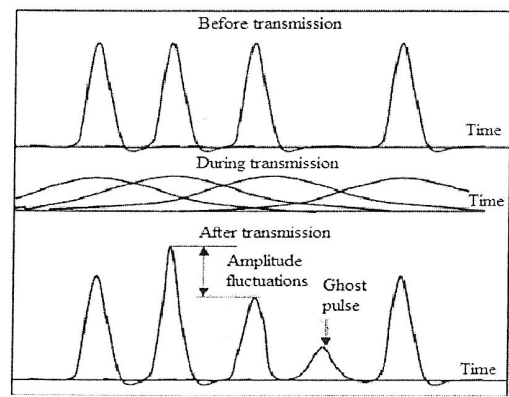

Fig. 5: The soliton pulses before transmission and after exceeding $250 \mathrm{~km}$ transmission.

Fig. 6 shows a 29.3 ps pulse of an actively mode-locked fiber laser with $5 \mathrm{GHz}$ repetition rate and an output peak power of $16 \mathrm{~mW}$. This pico-second pulse has some negative chirping that can be utilized to generate a soliton pulses. The pulse can be made nearly chirp-free by passing it through a dispersion compensation fiber (DCF) with a normal group velocity dispersion, GVD $\left(\beta_{2}>0\right)$, which allows for pulse compression. Fig. 7 shows the compressed pulse which is then amplified by a suitable amplifier.

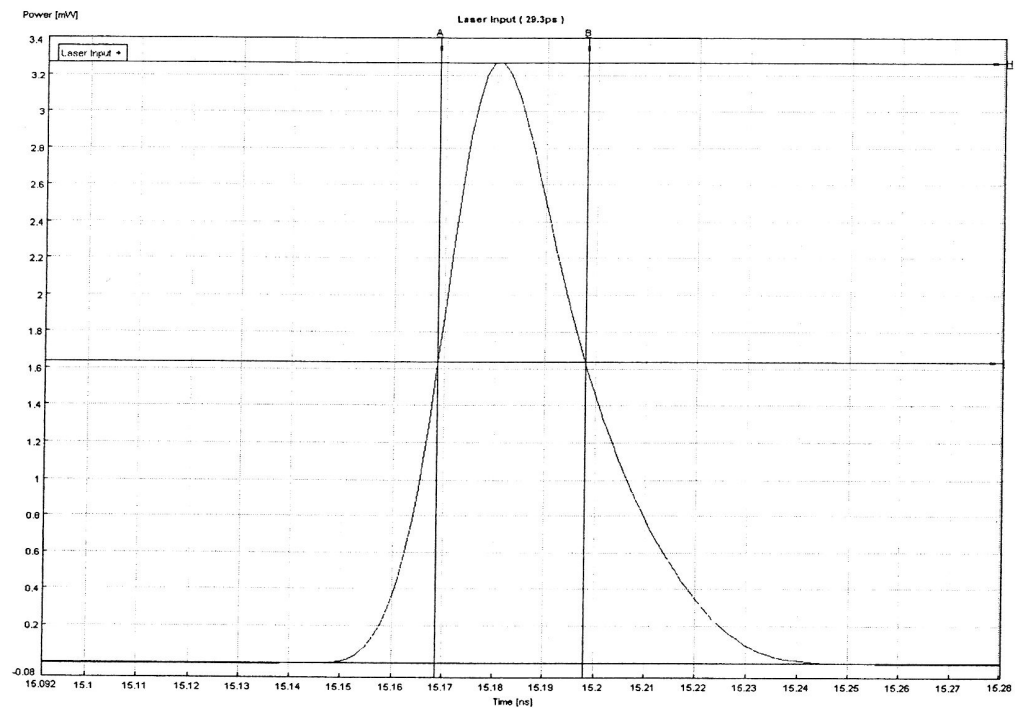

Fig. 6: The actively mode-locked laser output (29.3ps). 


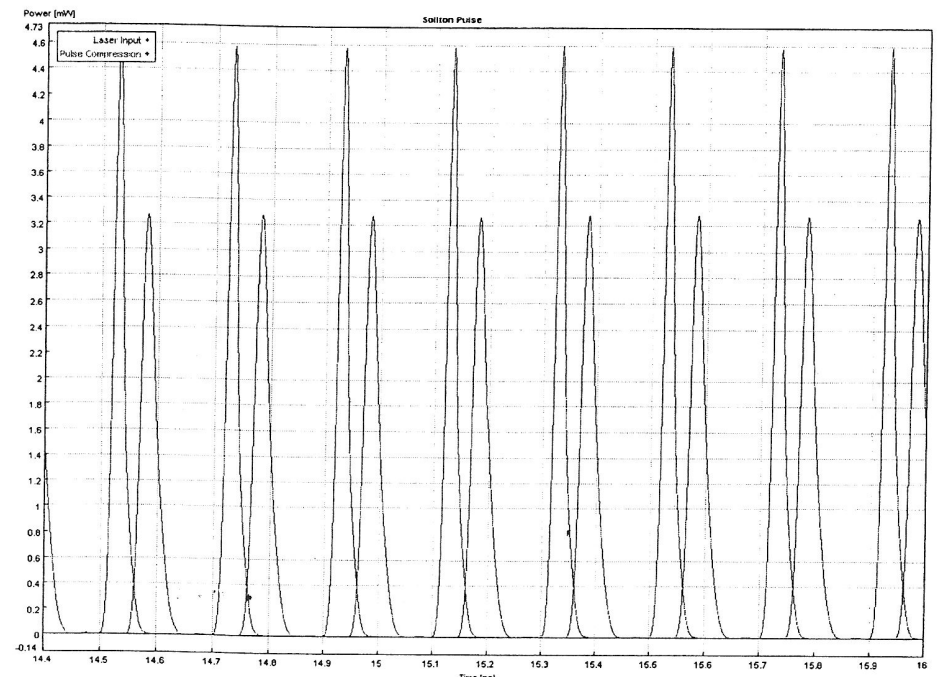

Fig. 7: The comparison between soliton laser input (29.3ps) and soliton compressed pulse (19.7ps).

As the pulses propagate, self-phase modulation (SPM) produces a positive chirp that puts low frequencies in the back of the pulse and high frequencies in the front. Positive dispersion (negative GVD) in a standard mode fiber, which is set to $+6 \mathrm{ps} / \mathrm{nm} / \mathrm{km}$, produces speed-up of the low frequencies and a slowdown of the high frequencies. The high frequencies in the back of the pulse fall further back and the low frequencies in front run further away. Hence the presence of SPM will cause faster disintegration of the pulse profile [4].

The system appears to be limited in maximum distance, and an increasingly narrow range of tolerable pulse widths. In order to improve these features, the approach that has been considered here was to reduce the average dispersion and optical power. Dispersion compensation is simple and effective; however it requires additional fiber length, greater optical gain (which may lead to additional amplifier noise), and a reasonable degree of accuracy. An alternative to these techniques is to make use of the nonlinearity of the optical fiber to counteract the dispersion. The $22 \mathrm{~km}$ standard mode fiber of $+6 \mathrm{ps} / \mathrm{nm} / \mathrm{km}$ and the $500 \mathrm{~m} \mathrm{DCF}$ of $-90 \mathrm{ps} / \mathrm{nm} / \mathrm{km}$ are found to counteract each other reasonably well and yield an average dispersion of $4 \mathrm{ps} / \mathrm{nm} / \mathrm{km}$. The average dispersion of the amplifier link, with the addition of the compensating fiber is found by considering the total dispersion across the link, as shown in Fig. 8, and utilizing the values shown in Fig. 9.

The introduction of a section of dispersion compensating fiber immediately before each amplifier is found to reduce the average dispersion. The reduction of the dispersion in this manner will result in an increase of the total transmission distance, and hence extends the range of tolerable pulse widths and reduces the average power requirement.

Fig. 10 and Fig. 11 show the shape and pulse width of a 22 ps output soliton propagating along a $22 \mathrm{~km}$ and $248 \mathrm{~km}$ fiber respectively. It can be noticed that pulse shape is unchanged. 


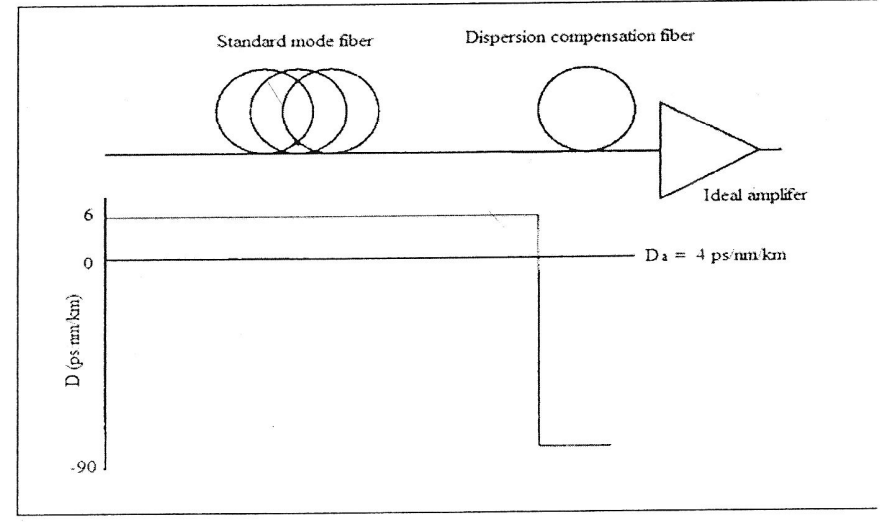

Fig. 8: Schematic diagram of the dispersion compensating and the variation of dispersion with distance over one transmission link.

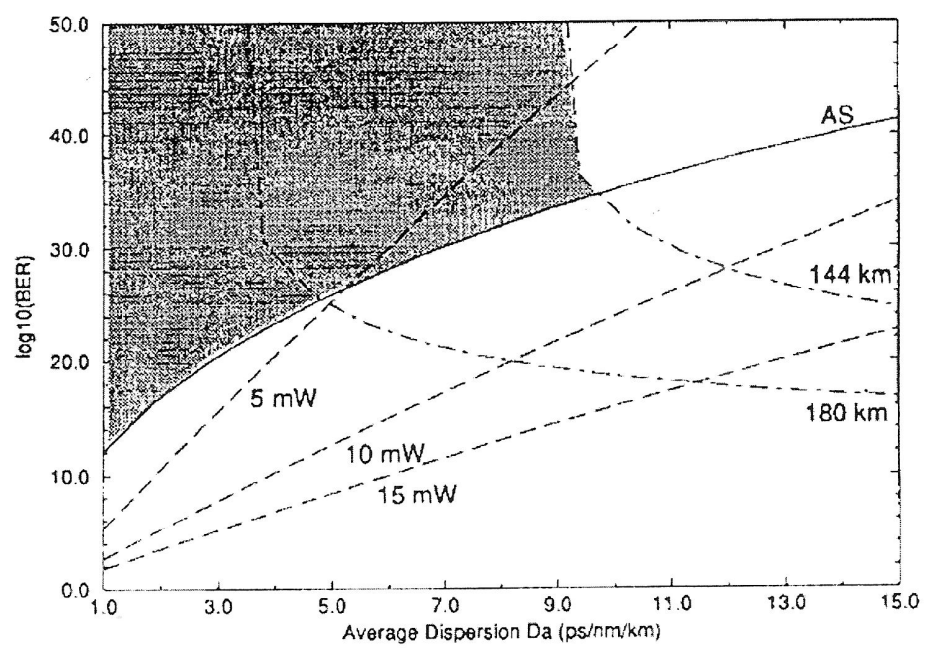

Fig.9: Dispersion compensation design diagram 


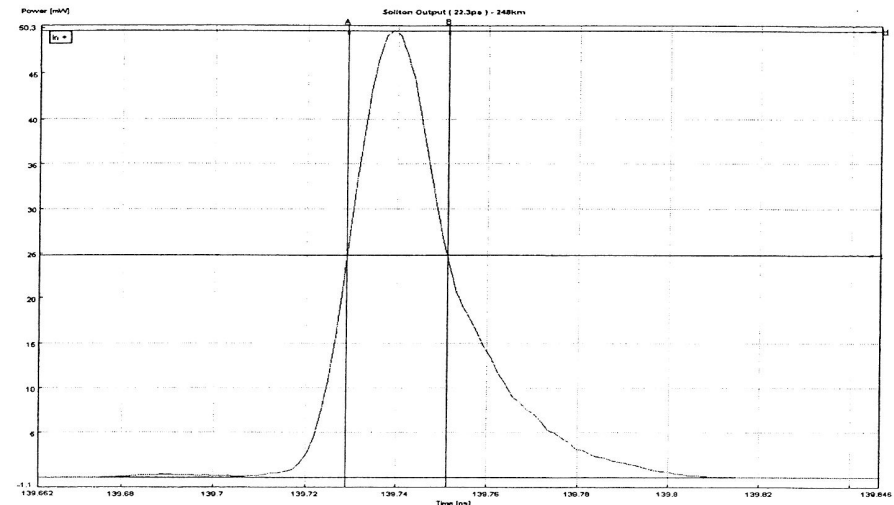

Fig. 10: Soliton output (22 km).

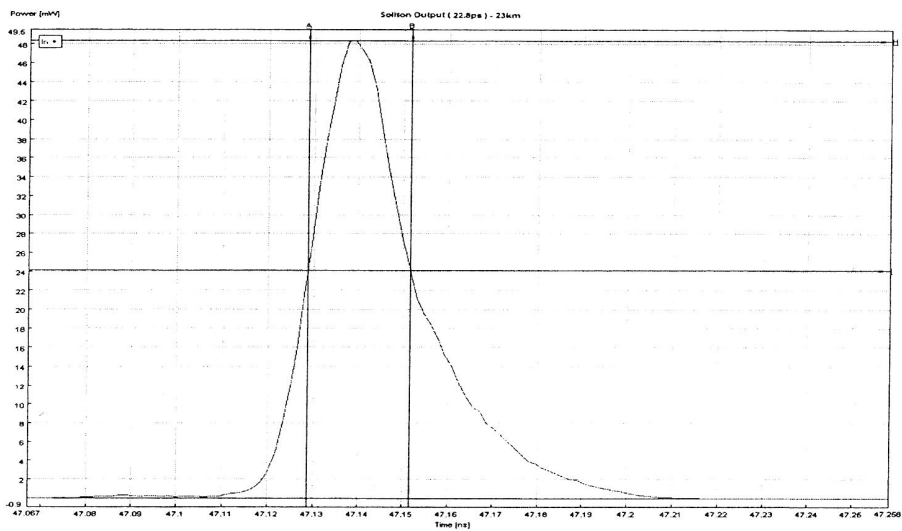

Fig. 11: Soliton output $(248 \mathrm{~km})$.

\section{CONCLUSION}

The transmission performance is improved by the insertion of the dispersion compensation elements. Reducing the dispersion before each amplifier section reduces the perturbations and improves the propagation of the solitons. It is predicted that solitons transmitted in long-haul systems will go beyond $250 \mathrm{~km}$ if the average optical powers is increased to more than $11 \mathrm{~mW}$. The analysis also suggests that the use of solitons will not only be desirable in future, but may become the only viable solution to meet the challenge. The choice of the $1550 \mathrm{~nm}$ minimizes the material loss, and hence reduces the required optical power. Shorter amplifier spacing may be necessary for the soliton pulses to retain their shape. Theoretically speaking, reducing the average dispersion to less than $4 \mathrm{ps} / \mathrm{nm} / \mathrm{km}$ and increasing the power levels above that of the average soliton, may lead to propagation distances that reach thousands of kilometers. 


\section{REFERENCES}

[1] Y. Takushima, T. Douke, X. Wang and K. Kikuchi, "Dispersion Tolerance and Transmission Distance of a 40-Gb/s Dispersion Management Soliton Transmission System", Lasers and Electro-Optics Society 2000 Annual Meeting, LEOS 2000, $13^{\text {th }}$ Annual Meeting IEEE, Volume 1., pp 157 - 158, 13 - 16 Nov 2000.

[2] M. Suzuki., "Dispersion Managed Long-Haul Soliton Transmission System", Lasers and Electro-Optics Society 2000 Annual Meeting. LEOS 2000, $13^{\text {th }}$ Annual Meeting IEEE, Volume 1, pp 153 - 154, 13 - 16 Nov. 2000.

[3] T. Hirooka, T. Nakada, and A. Hasegawa, Life Fellow IEEE,. "Feasibility of Densely Dispersion Managed Soliton Transmission at $160 \mathrm{~Gb} / \mathrm{s}$ ", Photonics Technology Letters, IEEE Volume 12, pp 633 - 635, Issue 6. June 2000.

[4] T. E. Murphy." 10-GHz 1.3-ps Pulse Generation Using Chirped Soliton Compression in a Raman Gain Medium,. Photonic Technology Letters, IEEE Volume 14, pp 1424 - 1426, Issue 10 Oct 2002

[5] G. Keiser, "Optical Fiber Communications" ch.12, $3^{\text {rd }}$ ed, pp 505-513, McGraw Hill, 2000 .

[6] M. Zafrullah, M. Waris, and M. K. Islam, "Simulation and Design of EDFAs for LongHaul Soliton Based Communication Systems", Communications 2002. APCC 2003, The $9^{\text {th }}$ Asia-Pacific Conference on Communications, Vol. 1, pp 25-27, $21-24$ Sept 2003.

[7] L. D. Coelho, Camelo J. A. Bastos-Filho and Joaquim F. Martins-Filho, "160 Gbit/s Soliton Transmission in the S and C Bands, Microwave and Optoelectronics Conference, 2003 IMOC 2003, Proceedings of the 200, SBMO/IEEE MTTS International, Volume 1, pp $245-249,22-23$ Sept 2003

[8] P. Kaewplung and T. Vijitrmekhtong, "Ideal Soliton Transmission In Lossy Fiber", Comunications and Information Technology 2004, ISCIT 2004, IEEE International Symposium on Vol. 2, pp $884-889,26-29$, Oct 2004. 